\title{
TP53 mutations in epithelial ovarian cancer
}

\author{
Yu Zhang ${ }^{1}$, Lan Cao ${ }^{1}$, Daniel Nguyen ${ }^{2}$, Hua Lu ${ }^{2}$ \\ ${ }^{1}$ Department of Gynecology and Obstetrics, Xiang-Ya Hospital, Central South University, Changsha 410008, China; ${ }^{2}$ Department of Biochemistry \\ and Molecular Biology, Tulane Cancer Center, Tulane University School of Medicine, New Orleans, LA, USA \\ Contributions: (I) Conception and Design: All authors; (II) Administrative support: Department of Gynecology and Obstetrics, Xiangya Hospital, \\ Central South University, Changsha, China; Department of Biochemistry and Molecular Biology, Tulane Cancer Center, Tulane University School \\ of Medicine, New Orleans, LA, USA; (III) Provision of study materials or patients: None; (IV) Collection and assembly of data: None; (V) Data \\ analysis and interpretation: None; (VI) Manuscript writing: All authors; (VII) Final approval of manuscript: All authors. \\ Correspondence to: Yu Zhang. Department of Gynecology and Obstetrics, Xiangya Hospital, Central South University, 87 Xiangya Road, Changsha \\ 410008, China. Email: cszhangyu@126.com; Hua Lu. Department of Biochemistry and Molecular Biology, Tulane Cancer Center, Tulane University \\ School of Medicine, New Orleans, LA 70112, USA. Email: hlu2@tulane.edu.
}

\begin{abstract}
Genomic sequencing analyses of a variety of human cancers have revealed that massive mutations of cancer-relevant genes are the major alterations in cancerous cells, and their mutation frequencies or rates are highly associated with the development, progression, metastasis, and drug resistance of cancers as well as their clinical outcomes and prognosis. One predominant genetic alternation in human epithelial ovarian cancer (EOC) is the mutation of TP53 that encodes the tumor suppressor p53 protein. This essay will review the most recent progress in understanding the role of TP53 mutations in development, progression, and metastasis of EOC, and discuss the potential of TP53 mutations as diagnostic and prognostic biomarkers as well as therapeutic targets for EOC.
\end{abstract}

Keywords: TP53 mutation; epithelial ovarian cancer (EOC); biomarkers; prognosis; chemoresistance; therapeutic target

Submitted May 25, 2016. Accepted for publication Jun 21, 2016.

doi: $10.21037 /$ tcr.2016.08.40

View this article at: http://dx.doi.org/10.21037/tcr.2016.08.40

\section{Introduction}

Epithelial ovarian cancer (EOC) is the most lethal gynecologic malignancy and the fifth most common cause of cancer-related death in women. The estimated annual incidence of this disease worldwide is 239,000 individuals, with approximately 152,000 deaths for the year 2012 (1). In China, EOC is also one of the top ten most common cancers in the female population. In 2011, the total ovarian cancer cases in China were 45,233 , and the incidence was $6.89 / 10^{5}$, which accounts for $3.11 \%$ of all female cancer patients (2). The risk for ovarian cancer increases with age. EOC is more common in women who have been through menopause (usually over the age of 50), although it can affect women of any age. The past several decades have witnessed both declining incidence and age adjusted cancer death rates for ovarian cancer. Significant advances in the understanding of the natural history of the disease and thorough initial staging, along with surgical and chemotherapeutic management, have improved the shortterm course of ovarian carcinoma. Recent statistics for cancer incidence and cancer death rates show that EOC death rates have declined by $14 \%$. The overall five-year survival rate for ovarian cancer has improved from $36 \%$ to $44 \%$. Among breast, ovarian, colorectal, and prostate cancers, EOC is the only one whose incidence rate declined more significantly than its death rate, even though progress in ovarian cancer research is far behind the other three (3). Despite these improvements, most of the EOC patients relapse after primary treatment and succumb to disease progression, and thus, EOC, particularly high-grade serous ovarian carcinoma (HGSOC) with a 10-year survival rate of only $15 \%$ (4), has been one of the more difficult cancers to treat. However, over the past decade, several breakthroughs 


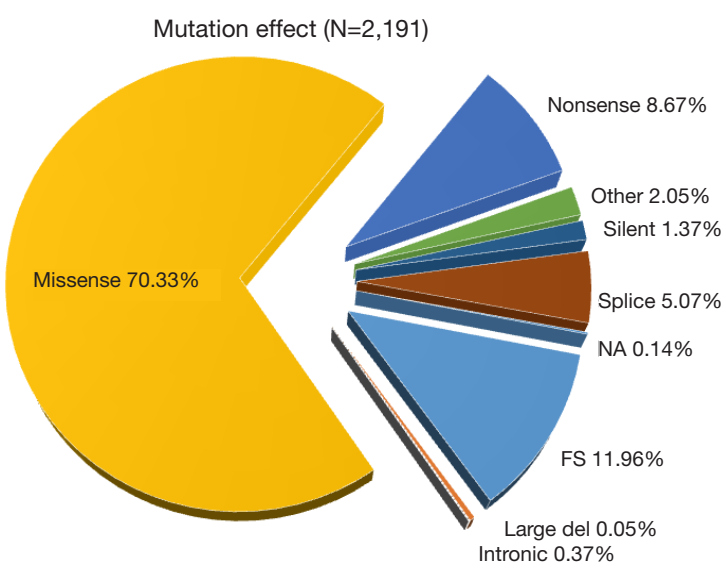

Figure 1 Percentage of the different types of TP53 somatic mutations in human ovarian cancers. FS, frameshift; NA, not applicable/not available. Data from the IARC TP53 Database (http://www-p53.iarc.fr/).

have been made to advance the diagnosis and treatment of EOC. In light of the heterogeneous histopathology and genetic mutations of EOC, EOC has been basically classified into Type I (low-grade tumors harboring BRAF, K-RAS and PTEN mutations) and Type II tumors (high-grade tumors characterized by TP53 mutations) (5). HGSOC accounts for $67 \%$ of all ovarian cancers and is the most aggressive subtype. A main characteristic of HGSOC is that all of the tumors bear p53 mutations $(6,7)$.

This review focuses on the clinical significance of TP53 mutations in EOC. We will describe the roles of mutant $\mathrm{p} 53 \mathrm{~s}$ in EOC carcinogenesis, their potential for the development of targeted therapy against EOC, and their prospects as potential biomarkers in screening and prognosis of EOC.

\section{Functions and mutations of p53}

p53 is a nuclear transcriptional regulator that participates in multiple cellular processes. By binding to DNA, p53 controls the expression of hundreds of target genes in order to maintain homeostasis and genome integrity. p53 can activate DNA repair proteins when DNA has sustained damage, arrest cell growth by holding the cell cycle at the G1/S transition, allowing DNA repair, and initiate apoptosis if DNA damage proves to be irreparable. The p53 protein contains four functional domains: an $\mathrm{N}$-terminal transcriptional activation domain, a central sequence-specific DNA binding domain, a tetramerization domain, and a C-terminal regulatory domain. In addition to transcriptional activating properties, transcriptional repression has been also ascribed to p53, although binding sites in its down-regulated target genes are less well characterized (8).

Numerous stimuli have been demonstrated to activate p53, including UV or gamma irradiation-induced DNA damage, inappropriate proto-oncogene activation, mitogenic signaling, ribosomal or nucleolar stress, and hypoxia (9-14). Once activated, p53 will induce cell cycle arrest, senescence, differentiation, apoptosis, or ferroptosis, depending upon the cellular context, by promoting the expression of a variety of genes crucial for the aforementioned cellular activities (15-17). For instance, p53 induces the expression of p21, which is responsible for $\mathrm{p} 53$-dependent cell cycle arrest at the G1 phase and senescence (18), while it induces the expression of Puma, Bax, or miR-34, which are critical for $\mathrm{p} 53$-dependent apoptosis (19-21). A recent study showed that $\mathrm{p} 53$ also induces ferroptosis, a special type of cell death caused by reactive oxygen species, by activating the expression of SLC7A11, a key component of the cystine/glutamate antiporter (15). Therefore, it is through transcriptional activity that p53 executes its anti-tumor functions in cells. These cellular anti-tumor functions of $\mathrm{p} 53$ could be utilized for the development of anticancer therapy.

Because of its essential role in tumor suppression, p53 is unsurprisingly found to be frequently mutated in cancer, as more than $50 \%$ of all types of human cancers have TP53 mutations. Over 36,000 TP53 mutations have been reported, and approximately $80 \%$ of $\mathrm{p} 53$ mutations are missense mutations with amino acid substitutions (22). There are 2,329 kinds of TP53 mutations that have been identified in human ovarian cancers through analysis of the IARC TP53 Database (http://www-p53.iarc.fr/), 70\% of which are missense mutations with few functionally similar to their wild type counterpart (Figure 1). Although TP53 point mutations, including those found in human ovarian cancer, are detected in most of the amino acid codons in this gene, $\sim 80 \%$ of them are clustered between exons 5 and 8 , which encode the highly conserved DNA binding domain of the p53 protein (Figure 2). Since p53 acts as a homotetrameric transcriptional factor, its mutations lead to three different phenotypes: loss-of-function (LOF), dominant-negative (DN), and gain-of-function (GOF). LOF is one main outcome of all of the p53 mutations, leading to the loss of the functions that wild type (WT) p53 possesses. These mutated $\mathrm{p} 53$ proteins are often very stable in cancer cells because they are unable to induce the expression of MDM2 and MDMX, the two physiological 
feedback inhibitors of $\mathrm{p} 53$, which form a complex to mediate $\mathrm{p} 53$ 's proteasomal turnover, as MDM2 possesses intrinsic E3 ubiquitin ligase activity (23-25). Also, missense mutations of $\mathrm{p} 53$ often dominantly negate the function of WT $\mathrm{p} 53$ because the former can form a heterotetramer with the latter, rendering the latter to lose its WT transcriptional activity. Interestingly, missense mutations of p53, particularly those so-called hot spot mutants (HSMs), such as R175H, G245S, R248W, R249S, R273C, R273H

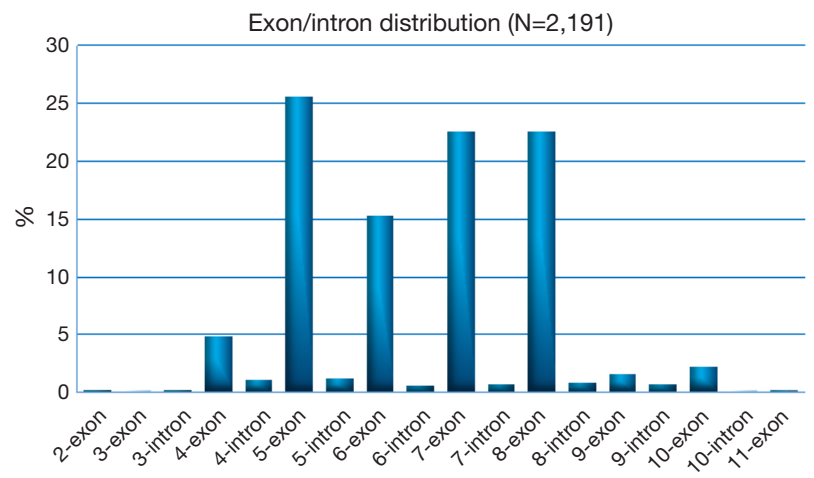

Figure 2 Percentage of somatic mutations in each exon or intron of TP53 in human ovarian cancers. Data obtained from the IARC TP53 Database (http://www-p53.iarc.fr/). or $\mathrm{R} 282 \mathrm{~W}$, though retaining partial activity ranging from 0 to $20 \%$ compared with the wild-type protein $(26,27)$, often acquire novel oncogenic functions and are appropriately named GOF mutants (28). In the coding sequence of TP53, the positions of somatic point mutations are very variable (Figure 3). On one hand, either loss of WT p 53 function, or the inability to activate $\mathrm{p} 53$, often severely compromises the capacity of cells to inhibit cellular proliferation and growth. Thus, the inactivation of the $\mathrm{p} 53$ pathway through either genetic mutations or functional inhibition will disable cells from protecting themselves against carcinogenesis. On the other hand, some missense mutations of $\mathrm{p} 53$ can even promote cancer cell proliferation, tumorigenesis, metastasis, and drug resistance through their new GOFs.

In sum, TP53 mutations are the most common genetic events that occur at a single gene in sporadic human EOC. The majority of HGSOCs harbor inactive p 53 molecules because of single genetic mutations. Therefore, p53 plays a critical role in preventing and inhibiting the development and progression of EOCs.

\section{Role of p53 mutations in cause and development of EOC}

The causes of human cancer are diverse, but malignant

A

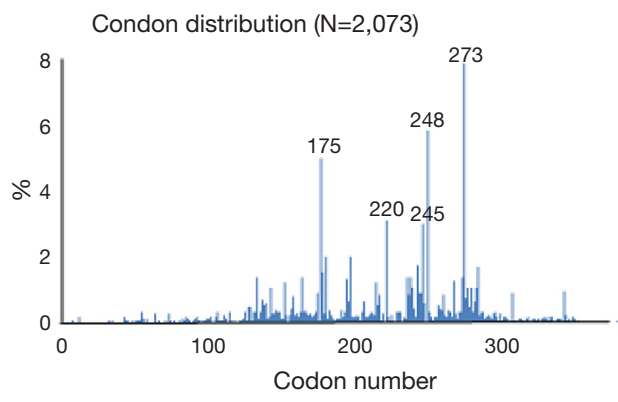

B

\begin{tabular}{|c|c|c|c|c|c|c|c|}
\hline \multicolumn{2}{|l|}{$\mathrm{N}$-terminal } & \multicolumn{3}{|c|}{ Central core } & \multicolumn{3}{|c|}{ C-terminal } \\
\hline & & & 220 & & NLS & & ES \\
\hline $1-42$ & $63-97$ & & 98-292 & & $300-323$ & $324-355$ & $363-393$ \\
\hline $\begin{array}{l}\text { Transactivation } \\
\text { domain }\end{array}$ & $\mathrm{SH} 3$ & $\left.\right|_{175}$ & DNA-binding domain $\|_{24 \$}$ & 273 & $\begin{array}{l}\text { Tetrame } \\
\text { domain }\end{array}$ & ization & $\begin{array}{l}\text { C-termina } \\
\text { regulatory } \\
\text { domain }\end{array}$ \\
\hline
\end{tabular}

Figure 3 p53 is highly mutated in its central DNA-binding domain. (A) Frequency of somatic point mutations with 5 most frequent mutants of $\mathrm{p} 53$ found in human ovarian cancers. Y-axis represents the percentage of mutation rates, and X-axis indicates the codon number of TP53. $\mathrm{N}=2,073$; (B) functional domains of $\mathrm{p} 53$ and the locations of the 5 most frequent mutations found in human ovarian cancers. NLS, nuclear localization signal; NES, nuclear export signal. Data from the IARC TP53 Database (http://www-p53.iarc.fr/). Numbers represent the positions of amino acids in p53. 
transformation is invariably caused by genetic alterations that lead to malfunction of the cell cycle and disruption of cell death. Several genetic alterations involved in carcinogenesis include changes in the sequence of genes (mutations), gains (amplifications) or losses (deletions) in the number of copies of genes, and rearrangement and translocation of genes from their normal chromosomal locations. These alterations lead to activation of genes favorable for proliferation (oncogenes) and inactivation of genes unfavorable for proliferation (tumor suppressor genes). Disruption of DNA repair systems also often occurs in the process of malignant transformation and may lead to accelerated accumulation of genetic alterations. Because repair of genetic damage inhibits carcinogenesis, DNA repair genes are also considered tumor suppressors. Although all of these genetic events might be associated with the cause and development of EOC, the most frequently occurred genetic alteration in EOC is TP53 mutation, as briefly mentioned above. Thus, we would like to further discuss the role of mutant $\mathrm{p} 53 \mathrm{~s}$ in the cause and development of EOC in this section.

EOCs are classified into several types according to their cellular phenotypes, including serous (the most common type), mucinous, endometrioid, clear cell, and undifferentiated or unclassifiable cells. Regardless of the presenting histopathology, they are currently all treated in a similar way. At first, EOCs were thought to originate solely from the epithelial cells that cover the surface of the ovary. Within the last decade, evidence that EOCs, particularly HGSOCs, can arise from the epithelial secretory cells of the fimbriae, a component of the Fallopian tube $(29,30)$. That HGSOCs can develop from precursor serous lesions of Fallopian tubal epithelial origin is highly relevant, as restricting experimental modeling to epithelial cells of ovarian origin would limit our understanding of the initiating tumorigenic mechanisms that govern HGSOCs (31). Nevertheless, the causes and risk factors of EOCs remain incompletely understood. The risk of developing ovarian cancer is very low in young women and increases as women become older. More than $80 \%$ of EOCs occur in women over the age of 50 . Approximately $10 \%$ of EOCs are thought to be due to an inherited altered gene (germ line mutation). Pathogenic TP53 mutations have been identified in $97 \%$ of HGSOC cases. The analysis of somatic mutations of HGSOC showed the enrichment of TP53 mutations though the frequencies were different in different ethnic groups $(32,33)$. Missense mutations in TP53 were found to be the most frequent in ovarian cancers, and early stage cancers had a significantly higher rate of null mutations than late stage disease (34). Overall, p53 dysfunction rate approached almost $100 \%$ in confirmed cases of HGSOCs, with only a few HGSOC cases harboring an intact p53 pathway $(32,35)$.

TP53 mutations occur early in tumor evolution and may be driver events in ovarian carcinogenesis $(36,37)$. Combined analysis (38) with The Cancer Genome Atlas (TCGA) indicate that low and late stage HGSOCs have similar mutation and copy number profiles. The deleterious TP53 mutations are also verified as the earliest events, followed by deletions or loss of heterozygosity ( $\mathrm{LOH})$ of chromosomes carrying TP53, BRCA1, and BRCA2. Inactivation of homologous recombination appears to be an early event, as $62.5 \%$ of tumors showed a $\mathrm{LOH}$ pattern suggestive of homologous recombination defects. Furthermore, polymorphism of TP53 may be a potential genetic modifier for development of EOC $(39,40)$. Animal studies also demonstrated the importance of TP53 mutations in the development of EOC, as ovaries or fallopian tubes that harbor $\mathrm{p} 53$ mutations can develop into HGSOC in mice as well (41).

Whereas most tumor suppressors, such as APC, PTEN, and BRCA1, are deleted during tumor initiation and progression, missense mutations account for over $70 \%$ of all known p53 alterations $(42,43)$, with many occurring at specific "hot spot" residues in the DNA binding domain, suggesting a selective advantage for these mutants. Indeed, prevalent p53 missense mutations not only abrogate its tumor suppressive function, but also acquire a GOF that promotes cancer development and progression. Characteristics, such as resistance to chemotherapy, deregulation of metabolic pathways, increased invasive capabilities, and increased metastatic potential, have all been attributed to GOF p53 (44-46). Several novel pathways involving the GOF of mutant p53 have been proposed to be highly related to cancer progression. For instance, one study showed that established cell lines harboring specific p53 missense mutations prevalently found in cancers $(R 248 Q, R 249 S, R 273 H$, and $R 175 H)$ universally bound to a novel group of chromatin regulatory genes in a genome-wide manner, and that expression of this chromatin signature was responsible for cancer phenotype (47). Specifically, by binding to chromatin regulatory genes and upregulating their expression, including methyltransferases MLL1 and MLL2 as well as acetyltransferase MOZ, these p53 GOF mutants promoted genome-wide increases of histone methylation and acetylation. These modifications 
of histones might drive mutant p53-dependent EOC progression, as the same missense mutations are frequently found in HGSOC. Analysis of TCGA in this study also revealed a good correlation between specific upregulation of these genes and EOCs that harbor GOFp53 mutants. An additional example of GOF activity involves the interaction with $\mathrm{p} 53$ family members $\mathrm{p} 63$ and $\mathrm{p} 73$, both of which also serve as tumor suppressors (48). Mutant p53 can form a complex with phosphorylated Smad2 and p63, simultaneously blocking wild-type p63 transcriptional targets while activating oncogenic genes, such as SHARP1, Dicer, and Cyclin G2, which have been implicated in invasion and metastasis (49-51). Similarly, in a pancreatic cancer mouse model, mutant p53 can directly bind to p73, preventing $\mathrm{p} 73$-mediated inactivation of PDGF $\beta$, driving an invasive and metastatic phenotype (52).

Another possible mechanism underlying the development and progression of EOC is via protein aggregation (53-56). Interestingly, some missense mutant $\mathrm{p} 53 \mathrm{~s}$ induce structural changes that expose adhesive segments, which can co-aggregate with wild-type p53 or its analogs p63 and p73, thereby inducing a heat-shock response and causing a trans- or cis-DN effect on the activity of these WT p53 family members. This suggests a different mechanism for clonal selection of aggregating p53 mutants, and thus some EOCs with this aggregation phenotype could be considered an aggregationassociated disease. An interesting notion concerning GOF p53 mutants is that tumor cells may become "addicted" to mutant $\mathrm{p} 53$ expression, providing an opportunity for pharmacological intervention and inhibition, as discussed below (57).

Although some progress has been made in bettering our understanding of the role of mutant $\mathrm{p} 53 \mathrm{~s}$ in the development and progression of EOCs, this field still confronts a number of challenges. Hence, further studies are needed to elucidate detailed molecular and biochemical mechanisms underlying the role of p53 mutants in EOC development and progression.

\section{Mutant p53 as screening biomarker for EOC}

The high mortality rate of EOC is largely due to the fact that over $60 \%$ of ovarian cancers are diagnosed after distant metastasis, as $90 \%$ of women who die from serous EOC present with late stage disease. At the metastatic stage, the 5- and 10-year survival rate for the EOC patients drops to $<30 \%$ and $<15 \%$, respectively (58). Early symptoms of EOC often are either entirely absent or subtle to point of misdiagnosis, allowing tumorigenesis to advance unchecked. A lack of viable early detection strategies can be attributed to a relatively rudimental understanding of the initiating events and mechanisms that drive the development and progression of EOC. Apparently, if EOC is diagnosed earlier, the survival rate for the patients can be as high as $90 \%$ (58). Therefore, early diagnosis is critically important for the improvement of EOC mortality and morbidity as well as for better prognosis of EOC patients.

To achieve this goal, the field has been trying to identify biomarkers for EOC. Despite the identification of serum CA125 as a biomarker for ovarian cancer in 1983 (59), there still has not been any screening biomarkers recommended for use for the general population since then. A recent study (60) showed that a combination of four serum proteins, CA-125, HE4, CEA, and VCAM-1, increases the sensitivity and specificity of detecting stage I and II ovarian cancer to $86 \%$ at $98 \%$, respectively, but this remains to be confirmed in a blinded validation study using prediagnostic sera. Thus, biomarkers are desperately needed to complement CA-125. Since TP53 is highly mutated in EOC, several studies have tried to explore the possibility of developing mutant p53s as biomarkers for this type of cancer. Serologically detectable p53 auto-antibodies (p53-AAbs) are a product of a spontaneous and early humoral immune response of the host to the accumulation of an antigenic mutated p53 protein in tumor cells. In EOC patients, p53-AAbs are found in $20-40 \%$ of serum samples and are associated with advanced (FIGO III-IV) stages (61). One study (62) using ELISA found that antibodies against $\mathrm{p} 53$ are detected in the sera of $42 \%$ of patients with serous ovarian cancer at $92 \%$ specificity. Also, Meta-analysis (63) in two databases showed that there is a significant association of serum p53AAbs with increased risk of EOC. Furthermore, a recent study using high-density programmable protein microarrays (NAPPA) showed 5,177 full-length candidate antigens in sera obtained from patients with serous ovarian cancer, and among these antigens, three AAbs against p53, PTPRA, and PTGFR, respectively, displayed the area under the curve (AUC) ranging from 64-74\% across two independent serum screenings and two technologic platforms (slide microarrays and bead arrays) (64). Using the three AAbs (when at least two AAbs were positive), the group detected a sensitivity of $23.3 \%$ at $98.3 \%$ specificity for EOC. These biomarkers maintain sensitivity in the setting of false-negative CA-125 levels, and, unlike CA-125, maintain specificity even when compared to benign ovarian disease. Despite the low detectable rate of p53-AAbs in serum, another group 
described the combinational use of protein and peptide epitopes as a novel approach to identify AAbs more specific for ovarian cancer screening (65). This method could reduce the number of false-positive samples in the healthy population. Altogether, these encouraging studies suggest that p53-AAbs detected in patients' sera may be promising biomarkers in EOC screening.

Two recent studies further supporting the possibility of detecting mutant p53 as an EOC biomarker utilize next generation sequencing of TP53 exons. One approach sought to detect tumor cells in the vagina of women with serous ovarian cancer by analyzing TP53 mutations in DNA samples collected from vaginal tampons, a highly attractive proposition as the procedure is entirely non-invasive (66). Interestingly, by detecting rare mutant alleles in a mixture of mutant and wild-type DNA, this group detected TP53 mutations in $60 \%$ of patients who suffered of serous carcinoma without a history of tubal ligation. While TP53 mutations were only detectable in vaginal DNA obtained from patients with serous carcinoma with intact fallopian tubes, the DNA might come from ovarian cancer cells that survive through the fallopian tube and uterus and reach the cervix and vagina intact. In all of the patients with mutations detected in the tampon specimens, the tumors and the vaginal DNAs harbored the exact same TP53 mutations. The fractions of DNA derived from exfoliated tumor cells ranged from $0.01 \%$ to $0.07 \%$. Although promising, more studies are necessary to further validate this method and to identify a more precise detection rate. Nevertheless, once optimized, this low-cost, high-throughput "vaginal tampon", and noninvasive approach to identify TP53 mutations may be eventually developed into a useful tool for the diagnosis of EOC.

Another recent study provided a proof of principal investigation into employing duplex sequencing, an ultradeep sequencing technique, to detect mutant p53 in the peritoneal fluid of women with HGSOC, as metastasis of HGSOC frequently permeates the peritoneum $(67,68)$. Moreover, HGSOC cells are often discovered in the peritoneal fluid even in the absence significant metastasis, perhaps because, as previously discussed, HGSOCs can develop from serous lesions in the fallopian tube, a retroperitoneal structure in direct contact with the peritoneal cavity (69). Duplex sequencing applies random molecular tagging of both strands of DNA to detect mutations with frequencies of $>1 / 10^{7}$, a dramatic improvement over single-stranded molecular tagging of DNA, which carries a false positive mutation rate of
$<1 / 10,000$ (70). The study reported a detection of tumor DNA in women with HGSOC with $94 \%$ sensitivity, even in cases deemed difficult to detect clinically, and an ability to distinguish cancer from healthy controls with $82 \%$ sensitivity and $90 \%$ specificity. Interestingly, the authors also showed that low frequency TP53 mutations in the peritoneal fluid and blood were found in nearly all women, even the healthy control group, and increased with age and cancer. Previously, this phenomenon of identifying cancerdriver genes in physiologically normal tissue was shown in blood and skin, providing further evidence that duplex sequencing may allow for identification of pre-existing clones with malignant potential $(71,72)$. Although the invasiveness of peritoneal lavage renders peritoneal fluid ultimately an impractical screening option, the accuracy afforded by duplex sequencing can potentially be applied to detecting TP53 mutations in non-invasive procedures, such as pap smears (73) and, as previously described, vaginal tampons.

In summary, although the limitation of these studies is that all specimens are from women with advanced stage diseases, and the application of these approaches to early stage EOCs has not been tested, it is believed that continuation of this line of exploration would not only open a new direction of research on EOC, but also promisingly lead to an eventually useful detection kit or tool for early EOC diagnosis.

\section{p53 as a therapeutic target for EOC}

In EOC clinics, the standard therapy of combining platinum with paclitaxel has been used for almost twenty years. For advanced stage EOC, neoadjuvant chemotherapy (NACT) with interval debulking surgery and postsurgery chemotherapy, has been in clinical application, although its efficacy is still under evaluation. For advanced stage EOC, patients are treated with a combination of surgery and chemotherapy to reduce the risk of the cancer recurrence, or to shrink any remaining cancer that could not be removed during the operation. Currently, the most optimal strategy seems to begin with primary, aggressive debulking surgery of the tumor, followed by intraperitoneal injection of chemotherapy, as chemo-resistant cells may be more difficult to surgically remove if NACT is applied first (4). Although intraperitoneal injection is associated with high toxicity and low patient compliance, it has also been shown to result in better survival rates when compared to intravenous injection (74). Over the past 
decade or so, progress in the development of new therapy against EOC has been slow, as currently there are few therapeutic options, and the overall survival rate of HGSOC remains poor. However, because of the advanced knowledge about the molecular biology of this type of cancer, there are some encouraging examples of molecule-targeted therapies which promise to be more effective and to provide the basis for personalized treatment, such as the use of anti-angiogenic (VEGF antibodies, tyrosine kinase inhibitors and angiopoietin antagonists) and polyadenosine diphosphate-ribose polymerase (PARP) inhibitors for EOC treatment (75). Unfortunately, combinations of chemotherapy with the VEGF inhibitor bevacizumab or the PARP inhibitor olaparib have thus far been shown to only delay disease progression and have had no bearing on preventing recurrence or death $(76,77)$. Clearly, developing new and effective anti-EOC therapies is desperately needed for curing this type of cancer and improving EOC patients' survival rate.

As discussed above, TP53 mutations are the most frequently occurred events in human EOC. Thus, p53 has become an attractive target for the development of molecule-targeted therapies for this disease. Over the past decade or so, several different approaches have been designed to target $\mathrm{p} 53$, including conversion of mutant $\mathrm{p} 53$ to a form with WT properties (e.g., PRIMA-1 and PRIMA$1 \mathrm{MET}$ ), prevention of $\mathrm{p} 53$ degradation by MDM2/4 antagonists [e.g., nutlins (78)], gene therapy (a retroviral p53 expression vector into an orthotopic human tumor model), and exploitation of synthetic lethality gene (SLG) principle. Anti-mutant p53 compounds are potentially useful for cancers with a high prevalence of p53 mutations, such as HGSOC, triple-negative breast cancer, and squamous lung cancer (79). This idea has been tested in animal model systems, as restoring WT p53 function in animal models can regress cancer development and significantly extend periods of survival. Also, this strategy has stimulated a variety of approaches to reactivate p53 as an anti-cancer therapeutic strategy (80).

Among the small molecules that target mutant p53 involved in ovarian epithelial cancer, PRIMA-1MET (also known as APR-246, Aprea AB) is perhaps one of the most promising examples. Presently, systemic carboplatin combination chemotherapy with PRIMA$1 \mathrm{MET}$ is undergoing phase $\mathrm{Ib} / \mathrm{II}$ clinical trial in patients with recurrent HGSOC (Clinical-Trials.gov Identifier: NCT02098343). PRIMA-1MET is able to reverse the oncogenic properties of mutant $\mathrm{p} 53$. It inhibits the growth of several different types of mutant $\mathrm{p} 53$-expressing malignant cells in culture and of xenograft tumors in animal models with little toxicity to the animals tested (81). Remarkably, PRIMA-1MET induces the expression of a panel of WT p53 target genes, such as p21, Noxa, Puma, GADD45, specific caspases (caspase 2, 3 and 9) and MDM2, in cancer cells that harbor mutant p53 (79). PRIMA-1MET can restore the WT functions of mutant p53, inducing cell cycle arrest and triggering apoptosis associated with the upregulation of proapoptotic proteins, caspase- 3 activation, and PARP cleavage in cancer cells obtained from a number of the patients treated (81). Although PRIMA-1MET has been reported to convert mutant p53 into its WT form, it remains unclear whether its derivatives could bind to or alter the properties of other cellular molecules. Thus, further research is required to establish the target specificity of this drug and its clinical application for HGSOC.

An alternative approach to develop new anti-EOC therapy is to utilize the concept of synthetic lethality (SL), which occurs when two non-lethal mutations bestow lethality if they are simultaneously present in a cell. One previous example of SL is the use of PARP inhibitors in breast and ovarian cancer containing germ-line defects in BRCA1/2 genes $(82,83)$. The SL strategy may be utilized to develop a new therapy by co-targeting mutant p53 and another cancer-related gene, such as PARP, BRCA1 or BRCA2 $(84,85)$. There are several studies using SL in vitro and in animal models as an anticancer therapeutic (86-88), although this approach has not yet been brought to the stage of clinical trials for EOC.

The prion-like properties of mutant p53 aggregates also offer potential targets for the development of therapeutic intervention in malignant tumor diseases $(56,89)$, though there has not been a specific drug used in clinic. More studies are clearly necessary at molecular levels in order to develop a therapy targeting the aggregates of mutant $\mathrm{p} 53 \mathrm{~s}$. One such promising approach is the recently developed cell penetrating 17-residue peptide named ReACp53, which was designed to target residues on $\mathrm{p} 53$ prone to aggregation $(90,91)$. This study showed that primary cells taken from patients with HGSOC have cytosolic p53 aggregates, but when treated with ReACp53, p53 became disaggregated, localized to the nucleus, and regained wild-type function. This nuclear translocation corresponded with a reduction in known GOF activity and an induction in wild-type activity, such as cell cycle arrest and apoptosis, in vitro on all cells harboring mutant $\mathrm{p} 53$, but not WT p53, suggesting that 
ReACp53 may not target normal tissue. ReACp53's efficacy translated to an in vivo model in which intraperitoneal injection, the recommended method of delivery of chemotherapeutic agents to patients with HGSOC, causes cell death and reduction of organ implants in a disseminated disease model. Although it will remain challenging to select the most effective treatment strategy on the basis of tumor phenotype and evolving genotype, it is believed that with proper use of newly identified mutant $\mathrm{p} 53$-targeted drugs at the appropriate phase of EOC, optimum anti-cancer therapy will eventually emerge from continual investigation on mutant $\mathrm{p} 53 \mathrm{~s}$ and clinical trials targeting these mutants.

Because there are a variety of missense mutations in EOC, it has also been proposed to design a drug to specifically target each individual p53 mutant with the hopes of having a more specific effect on EOCs that harbor specific mutants, thereby overcoming any potential chemoresistance (92). Although this is an enchanting idea, achieving this goal would require a better understanding of the molecular and pathological pathways altered by each mutation of $\mathrm{p} 53$ in this aggressive disease. Also, this line of information will be certainly valuable for precision oncology or individualized medicine in the treatment of EOC in the future.

\section{p53 as prognostic marker of EOC}

\section{Correlations of TP53 mutation with chemoresistance}

Chemoresistance has been a major issue in ovarian cancer clinic. Although most of the ovarian cancer patients initially responded well to chemotherapy, they often relapsed due to drug resistance. Currently, there are no appropriate biomarkers that can be used to predict patient response to chemotherapy. With the growing use of NACT for advanced ovarian cancer in management of ovarian cancers, it is crucially important to identify post-NACT biomarkers for ovarian cancers and to evaluate their utility in prediction of response to therapy and prognosis. To do so, several studies have focused on investigating whether the expression of $\mathrm{p} 53$ and its mutations are correlated with chemoresistance of ovarian cancers largely because TP53 mutations are the most frequent genetic alterations in this type of disease.

Remarkably, ovarian cancer patients harboring different mutated TP53 show different chemotherapy resistances and survival outcomes. One study compared overall survival of ovarian cancers patients with different TP53 HSMs by analyzing the TCGA database, and also determined the effects of microtubule stabilizers, such as paclitaxel, epothilone B (epoB), or ixabepilone, on ovarian cancers cell growth in cultured cells after introducing each of the p53 HSMs into the cells (93). Their results showed that R248G confers chemoresistance and is not acetylated during epoB treatment, while $\mathrm{R} 273 \mathrm{H}$ demonstrated high MDR1 expression and resistance to paclitaxel. Optimally cytoreduced patients with codon R273, R248, or R175 HSMs, or any other TP53 mutation have different overall survivals for 84.1, 33.6, 62.1, and 44.5 months, respectively. Another study (94) investigated the association of TP53 K351N mutation with drug-resistance by allele specific real-time PCR and DNA sequencing analyses of tumor samples obtained from 153 patients with advanced EOC who received platinum-based chemotherapy. This study showed that TP53 K351N mutation is associated with induction of platinum resistance after NACT, and is an independent factor for shorter disease free survival in multivariate analysis. Reversing this phenomenon has presented a potential strategy for developing more effective treatment of relapsed EOCs, though quite challenging (95-97). One obvious approach as mentioned above is to combine cisplatin, a genotoxic drug that induces p53-dependent and independent apoptosis, with APR-246. This combination might be more suitable for EOC patients that harbor $\mathrm{p} 53$ mutations and are resistant to standard chemotherapy.

Another strategy is to utilize the concept of the aggregation property of mutant $\mathrm{p} 53$, as this property has been discovered in different types of cancers and may be responsible for impairing the normal transcriptional activation and pro-apoptotic functions of $\mathrm{p} 53$. In a unique population of HGSOC cancer cells with cancer stem cell properties, p53 protein aggregation has proved to be associated with $\mathrm{p} 53$ inactivation and platinum resistance by a recent study (98). When these cancer stem cells differentiated into their chemosensitive progeny, they lost tumor-initiating capacity and p53 aggregates. Moreover, this study demonstrated that the overexpression of a p53-positive regulator, p14ARF, inhibits MDM2-mediated p53 degradation and leads to an imbalance of p 53 turnover that promotes the formation of $\mathrm{p} 53$ aggregates in both in vitro and in vivo models. Furthermore, by employing two-dimensional gel electrophoresis and mass spectrometry, this group discovered that aggregated p 53 might function uniquely by interacting with proteins that are critical for cancer cell survival and tumor progression. The poor chemoresponse of a subset of HGSOC patients suggest p53 aggregation as a new biomarker for chemoresistance. 
Table 1 Association of TP53 mutation with clinic prognosis of human EOC

\begin{tabular}{llll}
\hline Year of publication & Method for mutation detection & Clinical outcomes & \\
\hline 2007 & IHC & Good outcome & $(102)$ \\
2008 & IHC ${ }^{\ddagger}$ and p53 sequencing & Good outcome & $(103)$ \\
2013 & IHC andp53 sequencing & Poor outcome & $(104)$ \\
2014 & OncoMap & No association & $(105)$ \\
2014 & IHC & No association \\
2015 & TCGA dataset & Poor outcome & $(106)$ \\
2003 & IHC and extracted DNA analysis & Good outcome & $(107)$ \\
\hline
\end{tabular}

${ }^{\dagger}$, overall survival, disease-free survival or drug response; ${ }^{\ddagger}$, immunohistochemistry; ${ }^{\S}$, in p53 null mutation; ${ }^{n}$, in p53 mutation without overexpression of p53.

Therefore, inhibiting p53 aggregation can reactivate p53 pro-apoptotic function and be crucial for improving EOC patients' responses to chemotherapy and consequently increasing their survival rate.

\section{Prognostic significance of TP53 in EOC}

Ovarian cancers are the most lethal gynecologic malignancy. With an overall survival at diagnosis of roughly $40 \%$ and the continuing development of new treatments, the medium-term outlook for women with ovarian cancer has been far better than it was previously (75). The highest fatality of ovarian cancers might be due to their late stage diagnosis and relapse with resistance to subsequent chemotherapy. Therefore, identification of prognostic biomarkers is important for prediction and improvement of EOC therapeutic outcomes and patients' survival rate. However, inconsistent results concluding the relationship of TP53 mutations with survival and drug response have led to a dispute over the prognostic and predictive values of TP53 status in cancer, and delayed the translation of the assessment of TP53 status into the clinic (99). With improvement in the experimental methods in the past decade, various studies in which TP53 status was accurately assessed by gene sequencing still showed controversial results (8). In the investigation of EOC, the general trend is that TP53 mutations are associated with poor survival, as well as with chemo-resistance (data summarized from the IARC TP53 Mutation Database). Many other studies also reported no significant relationship, and few showed opposite results $(100,101)$. However, data collected from the IARC TP53 Mutation Database is not updated (the up-to-date references was collected in 2004). Recently, more and more studies have accurately assessed the association of TP53 status with clinical prognosis in EOC, as listed in Table 1 (of note, only studies with cohorts of $>50$ patients in the past decade are considered here). These studies investigating different mutations detected by different methods, showed inconsistent results.

These collected studies in the past decade seemingly suggest that TP53 mutations might not serve as an ideal and useful biomarker for EOC prognosis, therefore more careful and systematic studies or Meta analyses are still necessary for better understanding of the prognostic significance of TP53 status in EOCs. Indeed, one comprehensive study (109) has examined the prognostic value of the expression of two p53 isoforms ( $\triangle 133 \mathrm{p} 53$ and $\Delta 40 \mathrm{p} 53)$ in patients with serous ovarian cancer, and indicated that $\Delta 133 \mathrm{p} 53$ constitutes an independent prognostic marker for good outcomes. Intriguingly, this co-relationship was observed in patients whose tumors expressed a mutant $\mathrm{p} 53$, suggesting that $\Delta 133 \mathrm{p} 53$ might suppress the actions of the mutant $\mathrm{p} 53$. These studies suggest that mutant p53 activity may be counteracted by $\Delta 133 \mathrm{p} 53$, which leads to more favorable prognosis in advanced serous ovarian carcinomas. The polymorphism of p53 mutants may also play a role in clinical outcomes. For example, a study assessed polymorphic variant of codon 72 by RFLP (restriction fragment-length polymorphism) and suggested that Pro/Pro genotype of 72 codon polymorphism could be an independent prognostic marker in ovarian carcinomas (110).

Other studies have been attempted to determine the role of serum p53-AAbs in prognosis of EOC $(111,112)$, which could serve as a biomarker for diagnosis of EOC as discussed above. To date, the prognostic significance of $\mathrm{p} 53$ - 
AAbs in EOC has been controversial. One recent MetaAnalysis (113) suggested that the presence of p53-AAbs in EOC patients' sera is significantly associated with a better overall survival only at multivariate analyses, suggesting that p53-AAbs have a limited clinical application. However, since the results of this work were based on only few investigations, more prospective studies are needed to verify the prognostic significance of p53-AAbs in EOCs.

\section{Conclusions}

Since p53 was discovered in 1979 , tremendous progress has been made in understanding of its role in different human cancers. However, the clinical application of TP53 mutational analysis has not yet been accomplished. In particular, TP53 mutations are highly associated with human EOCs, as TP53 mutations are identified in $97 \%$ of the most aggressive HGSOCs that account for more than $2 / 3$ of all ovarian cancers. The diagnostic, therapeutic, prognostic, and predictive values of TP53 mutations are still not fully explored despite recent progresses as described above. Also, the effect of specific TP53 mutations (especially GOF TP53 mutation) on EOCs remains to be systematically investigated. Despite of the slow progress in the p53-EOC field, it is now realized that it will be critically important to identify p53 as a biomarker for cancer risk, prognosis, and response to currently used therapy, and to determine the application of $\mathrm{p} 53$ as a potential target for the development of effective molecule-targeted therapies for EOCs in the near future.

\section{Acknowledgements}

Funding: Y Zhang was supported in part by Science and Technology Plan of Hunan Province, China (grants number 2013FJ4114). H Lu was supported in part by National Institution of Health-National Cancer Institute (grants number R01CA095441, R01CA172468, R01CA127724, R21CA190775, and R21CA201889).

\section{Footnote}

Conflicts of Interest: The authors have no conflicts of interest to declare.

\section{References}

1. Torre LA, Bray F, Siegel RL, et al. Global cancer statistics,
2012. CA Cancer J Clin 2015;65:87-108.

2. Chen $\mathrm{W}$, Zheng $\mathrm{R}$, Zeng $\mathrm{H}$, et al. Annual report on status of cancer in China, 2011. Chin J Cancer Res 2015;27:2-12.

3. Goff B. Measuring ovarian cancer care: why are we still failing? Gynecol Oncol 2015;136:1-2.

4. Narod S. Can advanced-stage ovarian cancer be cured? Nat Rev Clin Oncol 2016;13:255-61.

5. Shih IeM, Kurman RJ. Ovarian tumorigenesis: a proposed model based on morphological and molecular genetic analysis. Am J Pathol 2004;164:1511-8.

6. Cancer Genome Atlas Research Network. Integrated genomic analyses of ovarian carcinoma. Nature 2011;474:609-15.

7. Vang R, Levine DA, Soslow RA, et al. Molecular Alterations of TP53 are a Defining Feature of Ovarian High-Grade Serous Carcinoma: A Rereview of Cases Lacking TP53 Mutations in The Cancer Genome Atlas Ovarian Study. Int J Gynecol Pathol 2016;35:48-55.

8. Brosh R, Rotter V. When mutants gain new powers: news from the mutant p53 field. Nat Rev Cancer 2009;9:701-13.

9. James A, Wang Y, Raje H, et al. Nucleolar stress with and without p53. Nucleus 2014;5:402-26.

10. Brooks CL, Gu W. New insights into p53 activation. Cell Res 2010;20:614-21.

11. Meek DW, Anderson CW. Posttranslational modification of p53: cooperative integrators of function. Cold Spring Harb Perspect Biol 2009;1:a000950.

12. Dai C, Gu W. p53 post-translational modification: deregulated in tumorigenesis. Trends Mol Med 2010;16:528-36.

13. Zhou X, Liao JM, Liao WJ, et al. Scission of the p53MDM2 Loop by Ribosomal Proteins. Genes Cancer 2012;3:298-310.

14. Zhang Y, Lu H. Signaling to p53: ribosomal proteins find their way. Cancer Cell 2009;16:369-77.

15. Jiang L, Kon N, Li T, et al. Ferroptosis as a p53-mediated activity during tumour suppression. Nature 2015;520:57-62.

16. Chen J. The Cell-Cycle Arrest and Apoptotic Functions of 553 in Tumor Initiation and Progression. Cold Spring Harb Perspect Med 2016;6:a026104.

17. Rufini A, Tucci P, Celardo I, et al. Senescence and aging: the critical roles of p53. Oncogene 2013;32:5129-43.

18. el-Deiry WS, Tokino T, Velculescu VE, et al. WAF1, a potential mediator of $\mathrm{p} 53$ tumor suppression. Cell 1993;75:817-25.

19. Nakano K, Vousden KH. PUMA, a novel proapoptotic gene, is induced by p53. Mol Cell 2001;7:683-94.

20. Miyashita T, Reed JC. Tumor suppressor p53 is a direct 
transcriptional activator of the human bax gene. Cell 1995;80:293-9.

21. He L, He X, Lim LP, et al. A microRNA component of the p53 tumour suppressor network. Nature 2007;447:1130-4.

22. Leroy B, Fournier JL, Ishioka C, et al. The TP53 website: an integrative resource centre for the TP53 mutation database and TP53 mutant analysis. Nucleic Acids Res 2013;41:D962-9.

23. Haupt Y, Maya R, Kazaz A, et al. Mdm2 promotes the rapid degradation of p53. Nature 1997;387:296-9.

24. Kubbutat MH, Jones SN, Vousden KH. Regulation of p53 stability by Mdm2. Nature 1997;387:299-303.

25. Honda R, Tanaka H, Yasuda H. Oncoprotein MDM2 is a ubiquitin ligase E3 for tumor suppressor p53. FEBS Lett 1997;420:25-7.

26. Brázdová $M$, Navrátilová L, Tichý V, et al. Preferential binding of hot spot mutant p53 proteins to supercoiled DNA in vitro and in cells. PLoS One 2013;8:e59567.

27. Edlund K, Larsson O, Ameur A, et al. Data-driven unbiased curation of the TP53 tumor suppressor gene mutation database and validation by ultradeep sequencing of human tumors. Proc Natl Acad Sci U S A 2012;109:9551-6.

28. Freed-Pastor WA, Prives C. Mutant p53: one name, many proteins. Genes Dev 2012;26:1268-86.

29. Perets R, Wyant GA, Muto KW, et al. Transformation of the fallopian tube secretory epithelium leads to high-grade serous ovarian cancer in Brca; Tp53;Pten models. Cancer Cell 2013;24:751-65.

30. Ng A, Barker N. Ovary and fimbrial stem cells: biology, niche and cancer origins. Nat Rev Mol Cell Biol 2015;16:625-38.

31. Karst AM, Levanon K, Drapkin R. Modeling high-grade serous ovarian carcinogenesis from the fallopian tube. Proc Natl Acad Sci U S A 2011;108:7547-52.

32. Hayano T, Yokota Y, Hosomichi K, et al. Molecular characterization of an intact p53 pathway subtype in highgrade serous ovarian cancer. PLoS One 2014;9:e114491.

33. Ab Mutalib NS, Syafruddin SE, Md Zain RR, et al. Molecular characterization of serous ovarian carcinoma using a multigene next generation sequencing cancer panel approach. BMC Res Notes 2014;7:805.

34. Bernardini MQ, Baba T, Lee PS, et al. Expression signatures of TP53 mutations in serous ovarian cancers. BMC Cancer 2010;10:237.

35. Ahmed AA, Etemadmoghadam D, Temple J, et al. Driver mutations in TP53 are ubiquitous in high grade serous carcinoma of the ovary. J Pathol 2010;221:49-56.
36. Hoogstraat M, de Pagter MS, Cirkel GA, et al. Genomic and transcriptomic plasticity in treatment-naive ovarian cancer. Genome Res 2014;24:200-11.

37. Hirasawa A, Masuda K, Akahane T, et al. Family history and BRCA1/BRCA2 status among Japanese ovarian cancer patients and occult cancer in a BRCA1 mutant case. Jpn J Clin Oncol 2014;44:49-56.

38. Chien J, Sicotte H, Fan JB, et al. TP53 mutations, tetraploidy and homologous recombination repair defects in early stage high-grade serous ovarian cancer. Nucleic Acids Res 2015;43:6945-58.

39. Dholariya S, Mir R, Zuberi M, et al. Potential impact of (rs 4645878) BAX promoter -248G\&gt;A and (rs 1042522) TP53 72Arg\&gt;pro polymorphisms on epithelial ovarian cancer patients. Clin Transl Oncol 2016;18:73-81.

40. Xi Y, Liu C, Xin X. Association between a single nucleotide polymorphism in the TP53 region and risk of ovarian cancer. Cell Biochem Biophys 2014;70:1907-12.

41. Kim J, Coffey DM, Ma L, et al. The ovary is an alternative site of origin for high-grade serous ovarian cancer in mice. Endocrinology 2015;156:1975-81.

42. Muller PA, Vousden KH. p53 mutations in cancer. Nat Cell Biol 2013;15:2-8.

43. Soussi T, Lozano G. p53 mutation heterogeneity in cancer. Biochem Biophys Res Commun 2005;331:834-42.

44. Blandino G, Levine AJ, Oren M. Mutant p53 gain of function: differential effects of different p 53 mutants on resistance of cultured cells to chemotherapy. Oncogene 1999; 18:477-85.

45. Li R, Sutphin PD, Schwartz D, et al. Mutant p53 protein expression interferes with $\mathrm{p} 53$-independent apoptotic pathways. Oncogene 1998;16:3269-77.

46. Morton JP, Timpson P, Karim SA, et al. Mutant p5 3 drives metastasis and overcomes growth arrest/ senescence in pancreatic cancer. Proc Natl Acad Sci U S A 2010;107:246-51.

47. Zhu J, Sammons MA, Donahue G, et al. Gain-of-function p53 mutants co-opt chromatin pathways to drive cancer growth. Nature 2015;525:206-11.

48. Flores ER, Sengupta S, Miller JB, et al. Tumor predisposition in mice mutant for $\mathrm{p} 63$ and $\mathrm{p} 73$ : evidence for broader tumor suppressor functions for the p53 family. Cancer Cell 2005;7:363-73.

49. Adorno M, Cordenonsi M, Montagner M, et al. A Mutant-p53/Smad complex opposes p63 to empower TGFbeta-induced metastasis. Cell 2009;137:87-98.

50. Girardini JE, Napoli M, Piazza S, et al. A Pin1/mutant p53 axis promotes aggressiveness in breast cancer. Cancer Cell 
2011;20:79-91.

51. Su X, Chakravarti D, Cho MS, et al. TAp63 suppresses metastasis through coordinate regulation of Dicer and miRNAs. Nature 2010;467:986-90.

52. Weissmueller S, Manchado E, Saborowski M, et al. Mutant p53 drives pancreatic cancer metastasis through cell-autonomous PDGF receptor $\beta$ signaling. Cell 2014;157:382-94.

53. Silva JL, Rangel LP, Costa DC, et al. Expanding the prion concept to cancer biology: dominant-negative effect of aggregates of mutant p53 tumour suppressor. Biosci Rep 2013;33.

54. Xu J, Reumers J, Couceiro JR, et al. Gain of function of mutant p53 by coaggregation with multiple tumor suppressors. Nat Chem Biol 2011;7:285-95.

55. Gong H, Yang X, Zhao Y, et al. Amyloidogenicity of p53: A Hidden Link Between Protein Misfolding and Cancer. Curr Protein Pept Sci 2014. [Epub ahead of print].

56. Rangel LP, Costa DC, Vieira TC, et al. The aggregation of mutant p 53 produces prion-like properties in cancer. Prion 2014;8:75-84.

57. Kim MP, Zhang Y, Lozano G. Mutant p53: Multiple Mechanisms Define Biologic Activity in Cancer. Front Oncol 2015;5:249.

58. Siegel R, Naishadham D, Jemal A. Cancer statistics, 2013. CA Cancer J Clin 2013;63:11-30.

59. Bast RC Jr, Klug TL, St John E, et al. A radioimmunoassay using a monoclonal antibody to monitor the course of epithelial ovarian cancer. N Engl J Med 1983;309:883-7.

60. Yurkovetsky Z, Skates S, Lomakin A, et al. Development of a multimarker assay for early detection of ovarian cancer. J Clin Oncol 2010;28:2159-66.

61. Tsai-Turton M, Santillan A, Lu D, et al. p53 autoantibodies, cytokine levels and ovarian carcinogenesis. Gynecol Oncol 2009;114:12-7.

62. Anderson KS, Wong J, Vitonis A, et al. p53 autoantibodies as potential detection and prognostic biomarkers in serous ovarian cancer. Cancer Epidemiol Biomarkers Prev 2010;19:859-68.

63. Shi JX, Qin JJ, Ye H, et al. Tumor associated antigens or anti-TAA autoantibodies as biomarkers in the diagnosis of ovarian cancer: a systematic review with meta-analysis. Expert Rev Mol Diagn 2015;15:829-52.

64. Anderson KS, Cramer DW, Sibani S, et al. Autoantibody signature for the serologic detection of ovarian cancer. J Proteome Res 2015;14:578-86.

65. Arjomandi A, Delanoy ML, Walker RP, et al. A Novel Algorithm to Improve Specificity in Ovarian Cancer
Detection. Clinical Ovarian and Other Gynecologic Cancer 2015. [Epub ahead of print].

66. Erickson BK, Kinde I, Dobbin ZC, et al. Detection of somatic TP53 mutations in tampons of patients with high-grade serous ovarian cancer. Obstet Gynecol 2014;124:881-5.

67. Krimmel JD, Schmitt MW, Harrell MI, et al. Ultra-deep sequencing detects ovarian cancer cells in peritoneal fluid and reveals somatic TP53 mutations in noncancerous tissues. Proc Natl Acad Sci U S A 2016;113:6005-10.

68. Naora H, Montell DJ. Ovarian cancer metastasis: integrating insights from disparate model organisms. Nat Rev Cancer 2005;5:355-66.

69. Prat J, FIGO Committee on Gynecologic Oncology. Staging classification for cancer of the ovary, fallopian tube, and peritoneum. Int J Gynaecol Obstet 2014;124:1-5.

70. Fox EJ, Reid-Bayliss KS, Emond MJ, et al. Accuracy of Next Generation Sequencing Platforms. Next Gener Seq Appl 2014;1.

71. Martincorena I, Roshan A, Gerstung M, et al. Tumor evolution. High burden and pervasive positive selection of somatic mutations in normal human skin. Science 2015;348:880-6.

72. Schmitt MW, Fox EJ, Prindle MJ, et al. Sequencing small genomic targets with high efficiency and extreme accuracy. Nat Methods 2015;12:423-5.

73. Kinde I, Bettegowda C, Wang Y, et al. Evaluation of DNA from the Papanicolaou test to detect ovarian and endometrial cancers. Sci Transl Med 2013;5:167ra4.

74. Tewari D, Java JJ, Salani R, et al. Long-term survival advantage and prognostic factors associated with intraperitoneal chemotherapy treatment in advanced ovarian cancer: a gynecologic oncology group study. J Clin Oncol 2015;33:1460-6.

75. Jayson GC, Kohn EC, Kitchener HC, et al. Ovarian cancer. Lancet 2014;384:1376-88.

76. Pujade-Lauraine E, Hilpert F, Weber B, et al. Bevacizumab combined with chemotherapy for platinum-resistant recurrent ovarian cancer: The AURELIA open-label randomized phase III trial. J Clin Oncol 2014;32:1302-8.

77. Oza AM, Cibula D, Benzaquen AO, et al. Olaparib combined with chemotherapy for recurrent platinumsensitive ovarian cancer: a randomised phase 2 trial. Lancet Oncol 2015;16:87-97.

78. Carrillo AM, Hicks M, Khabele D, et al. Pharmacologically Increasing Mdm2 Inhibits DNA Repair and Cooperates with Genotoxic Agents to Kill p53-Inactivated Ovarian Cancer Cells. Mol Cancer Res 2015;13:1197-205. 
79. Duffy MJ, Synnott NC, McGowan PM, et al. p53 as a target for the treatment of cancer. Cancer Treat Rev 2014;40:1153-60.

80. Schmid BC, Oehler MK. New perspectives in ovarian cancer treatment. Maturitas 2014;77:128-36.

81. Zandi R, Selivanova G, Christensen CL, et al. PRIMA$1 \mathrm{Met} / \mathrm{APR}-246$ induces apoptosis and tumor growth delay in small cell lung cancer expressing mutant p53. Clin Cancer Res 2011;17:2830-41.

82. Bryant HE, Schultz N, Thomas HD, et al. Specific killing of BRCA2-deficient tumours with inhibitors of poly(ADPribose) polymerase. Nature 2005;434:913-7.

83. O'Connor MJ. Targeting the DNA Damage Response in Cancer. Mol Cell 2015;60:547-60.

84. Thompson JM, Nguyen QH, Singh M, et al. Approaches to identifying synthetic lethal interactions in cancer. Yale J Biol Med 2015;88:145-55.

85. Fang B. Development of synthetic lethality anticancer therapeutics. J Med Chem 2014;57:7859-73.

86. Tiong KL, Chang KC, Yeh KT, et al. CSNK1E/CTNNB1 are synthetic lethal to TP53 in colorectal cancer and are markers for prognosis. Neoplasia 2014;16:441-50.

87. Bitler BG, Aird KM, Garipov A, et al. Synthetic lethality by targeting EZH2 methyltransferase activity in ARID1Amutated cancers. Nat Med 2015;21:231-8.

88. Morandell S, Reinhardt HC, Cannell IG, et al. A reversible gene-targeting strategy identifies synthetic lethal interactions between MK2 and p53 in the DNA damage response in vivo. Cell Rep 2013;5:868-77.

89. Silva JL, De Moura Gallo CV, Costa DC, et al. Prion-like aggregation of mutant p53 in cancer. Trends Biochem Sci 2014;39:260-7.

90. Soragni A, Janzen DM, Johnson LM, et al. A Designed Inhibitor of p53 Aggregation Rescues p53 Tumor Suppression in Ovarian Carcinomas. Cancer Cell 2016;29:90-103.

91. Goldschmidt L, Teng PK, Riek R, et al. Identifying the amylome, proteins capable of forming amyloid-like fibrils. Proc Natl Acad Sci U S A 2010;107:3487-92.

92. Brachova P, Thiel KW, Leslie KK. The consequence of oncomorphic TP53 mutations in ovarian cancer. Int J Mol Sci 2013;14:19257-75.

93. Seagle BL, Yang CP, Eng KH, et al. TP53 hot spot mutations in ovarian cancer: selective resistance to microtubule stabilizers in vitro and differential survival outcomes from The Cancer Genome Atlas. Gynecol Oncol 2015;138:159-64.

94. Zhang GN, Liu H, Huang JM, et al. TP53 K351N mutation-associated platinum resistance after neoadjuvant chemotherapy in patients with advanced ovarian cancer. Gynecol Oncol 2014;132:752-7.

95. Li B, Gao Y, Rankin GO, et al. Chaetoglobosin K induces apoptosis and G2 cell cycle arrest through p53-dependent pathway in cisplatin-resistant ovarian cancer cells. Cancer Lett 2015;356:418-33.

96. Liu Q, Sui R, Li R, et al. Biological characteristics of Taxol-resistant ovarian cancer cells and reversal of Taxol resistance by adenovirus expressing p53. Mol Med Rep 2015;11:1292-7.

97. Mir R, Stanzani E, Martinez-Soler F, et al. YM155 sensitizes ovarian cancer cells to cisplatin inducing apoptosis and tumor regression. Gynecol Oncol 2014;132:211-20.

98. Yang-Hartwich Y, Soteras MG, Lin ZP, et al. p53 protein aggregation promotes platinum resistance in ovarian cancer. Oncogene 2015;34:3605-16.

99. Hainaut P, Wiman KG. editors. 25 Years of p53 Research. Springer Netherlands, 2007.

100. Niwa K, Itoh M, Murase T, et al. Alteration of p53 gene in ovarian carcinoma: clinicopathological correlation and prognostic significance. Br J Cancer 1994;70:1191-7.

101.Leitao MM Jr, Boyd J, Hummer A, et al. Clinicopathologic analysis of early-stage sporadic ovarian carcinoma. Am J Surg Pathol 2004;28:147-59.

102. Böhnke A, Jung J, Taubert H, et al. Both somatic and germline genetics of the TP53-pathway influence ovarian cancer incidence and survival. Verh Dtsch Ges Pathol 2007;91:233-42.

103. Bartel F, Jung J, Böhnke A, et al. Both germ line and somatic genetics of the p53 pathway affect ovarian cancer incidence and survival. Clin Cancer Res 2008;14:89-96.

104. Nadkarni NJ, Geest KD, Neff T, et al. Microvessel density and p53 mutations in advanced-stage epithelial ovarian cancer. Cancer Lett 2013;331:99-104.

105. Kim YM, Lee SW, Chun SM, et al. Analysis and comparison of somatic mutations in paired primary and recurrent epithelial ovarian cancer samples. PLoS One 2014;9:e99451.

106. Khandakar B, Mathur SR, Kumar L, et al. Tissue biomarkers in prognostication of serous ovarian cancer following neoadjuvant chemotherapy. Biomed Res Int 2014;2014:401245.

107. Brachova P, Mueting SR, Carlson MJ, et al. TP53 oncomorphic mutations predict resistance to platinumand taxane-based standard chemotherapy in patients diagnosed with advanced serous ovarian carcinoma. Int J 
Oncol 2015;46:607-18.

108. Havrilesky L, Darcy kM, Hamdan H, et al. Prognostic significance of $\mathrm{p} 53$ mutation and $\mathrm{p} 53$ overexpression in advanced epithelial ovarian cancer: a Gynecologic Oncology Group Study. J Clin Oncol 2003;21:3814-25.

109. Chambers SK, Martinez JD. The significance of p 53 isoform expression in serous ovarian cancer. Future Oncol 2012;8:683-6.

110.Malisic E, Jankovic R, Slavkovic D, et al. p53 gene mutations and codon 72 polymorphism in ovarian carcinoma patients from Serbia. J BUON 2010;15:101-6.

Cite this article as: Zhang Y, Cao L, Nguyen D, Lu H. TP53 mutations in epithelial ovarian cancer. Transl Cancer Res 2016;5(6):650-663. doi: 10.21037/tcr.2016.08.40
111. Salmena L, Shaw P, Fans I, et al. Prognostic value of INPP4B protein immunohistochemistry in ovarian cancer. Eur J Gynaecol Oncol 2015;36:260-7.

112.Dobrzycka B, Terlikowski SJ, Kinalski M, et al. Circulating free DNA and $\mathrm{p} 53$ antibodies in plasma of patients with ovarian epithelial cancers. Ann Oncol 2011;22:1133-40.

113. Garziera M, Montico M, Bidoli E, et al. Prognostic Role of Serum Antibody Immunity to p53 Oncogenic Protein in Ovarian Cancer: A Systematic Review and a MetaAnalysis. PLoS One 2015;10:e140351. 